\title{
Contemporary health care economics: an overview
}

\author{
Nancy McLaughlin, M.D., Ph.D., F.R.C.S.C., ${ }^{1}$ Michael K. Ong, M.D., Ph.D., ${ }^{2}$ \\ Victor Tabbush, Ph.D., ${ }^{3}$ Farhad Hagigi, Ph.D., ${ }^{3}$ and Neil A. Martin, M.D. ${ }^{1}$ \\ Departments of ${ }^{1}$ Neurosurgery and ${ }^{2}$ Internal Medicine, David Geffen School of Medicine at UCLA; \\ and ${ }^{3}$ UCLA Anderson School of Management, Los Angeles, California
}

\begin{abstract}
Economic evaluations provide a decision-making framework in which outcomes (benefits) and costs are assessed for various alternative options. Although the interest in complete and partial economic evaluations has increased over the past 2 decades, the quality of studies has been marginal due to methodological challenges or incomplete cost determination. This paper provides an overview of the main types of complete and partial economic evaluations, reviews key methodological elements to be considered for any economic evaluation, and reviews concepts of cost determination. The goal is to provide the clinician neurosurgeon with the knowledge and tools needed to appraise published economic evaluations and to direct high-quality health economic evaluations.
\end{abstract}

(http://thejns.org/doi/abs/10.3171/2014.8.FOCUS14455)

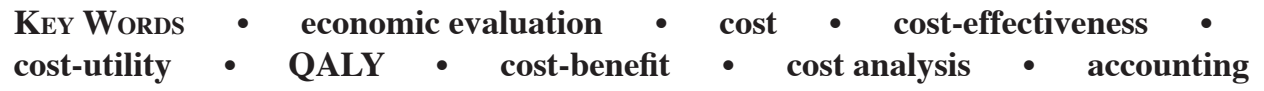

$\mathrm{E}$ CONOMIC evaluations were developed more than 50 years ago to assist those responsible for allocating resources to prioritize alternatives with the maximum benefit at the lowest cost, as health care resources were, and remain, scarce. ${ }^{27}$ Economic evaluations provide a decision-making framework in which advantages (outcomes achieved) and disadvantages (costs to achieve the outcomes) are assessed for each alternative. ${ }^{27}$ They help assess whether the additional benefits are worth the additional costs.

Over the past 2 decades, there has been growing interest in health economic evaluation and cost analysis concerning procedures and resources used in the care of neurosurgical patients. The absolute number of studies including among its key words "cost," "cost minimization," "cost-effectiveness," "cost-utility," "cost-benefits," "cost analysis," cost containment," or "economic evaluation" has increased over the past decade, and more rapidly so in the last few years. Despite this manifestly growing interest, there have been important gaps in the quality of published health economic evaluations as they pertain to the selection of evaluation and key methodological elements. ${ }^{17}$ Although clinicians may have been acquainted

Abbreviations used in this paper: $\mathrm{CBA}=$ cost-benefit analysis; $\mathrm{CEA}=$ cost-effectiveness analysis; $\mathrm{CMA}=$ cost minimization analysis; $\mathrm{CUA}=$ cost-utility analysis; EQ-5D = EuroQol 5 Dimension; HCUP = Healthcare Cost and Utilization Project; ICER = incremental cost-effectiveness ratio; NIS = Nationwide Inpatient Sample; QALY = quality-adjusted life year. with outcome determination, cost determination has been and remains challenging. Costs have been estimated using financial reports and national databases, most commonly capturing only hospital-related costs. But in the majority of cases, other surrogates have been used including charges (billing), reimbursement (Medicare allowable payment amounts, and home-engineered methods to estimate costs).

In this paper we provide an overview of the main types of complete economic evaluations as well as cost analysis, and we review key methodological elements to be included in economic evaluations (and cost analysis) and concepts of cost determination. The goal is to provide the clinician neurosurgeon with the knowledge and tools needed to appraise published economic evaluations and to direct high-quality health economic evaluations. The importance of establishing a collaborative relationship among finance specialists (decision support team), economists, performance excellence experts, and clinicians cannot be overstressed to complete a robust analysis.

\section{Overview of Complete Economic Evaluations}

Four main types of complete economic evaluations are currently used: cost minimization analysis (CMA), cost-effectiveness analysis (CEA), cost-utility analysis (CUA), and cost-benefit analysis (CBA). The most critical initial step is to formulate the study question; this will guide selection of the appropriate type of economic evaluation. Reviewing in depth each type of economic 
evaluation is beyond the scope of this overview. A concise description of each type follows. Table 1 presents a summary of the 4 main economic evaluations.

\section{Cost Minimization Analysis}

In a CMA, the alternative options are known to have the same effectiveness (efficacy or clinical outcome). ${ }^{11}$ The costs of the 2 or more procedures or programs under consideration are compared to establish the lowest cost alternative. It is essential that the same or similar effectiveness has been demonstrated by a clinical study that is specifically designed to assess the equivalence of treatments. ${ }^{4}$

As an example, Heaney and colleagues performed an economic evaluation of the use of carbamazepine, lamotrigine, phenytoin, and valproic acid as the initial treatment in adults with newly diagnosed epilepsy. ${ }^{10}$ Studies had demonstrated that the drugs had equivalent effectiveness in controlling seizures, and therefore the authors performed a CMA to compare only the direct costs of similarly effective therapies. Published CMAs are rare in the neurosurgical literature as outcomes are most frequently different between two alternative options.

\section{Cost-Effectiveness Analysis}

In CEA, the clinical outcomes and costs of each procedure or program are assessed and compared. The costs are expressed in monetary units and the outcomes in nonmonetary natural units, common to all alternatives. ${ }^{28}$ Examples of clinical outcomes include symptom-free days, specific clinical parameters (e.g., remission rates in functional pituitary surgery), hospital days prevented, and life years gained. Identification of the most appropriate outcomes to serve as effectiveness end points is crucial. Selected outcomes often provide a narrow view, focusing on one condition or specific care pathway, and limiting the use of CEA to guide allocation of resources across different health programs. ${ }^{7}$

Karinen and colleagues assessed the cost-effectiveness of nimodipine administration in patients who presented with a subarachnoid hemorrhage and underwent aneurysm clipping. ${ }^{20}$ The authors used direct costs for the hospitalization, and the nonmonetary clinical outcome was measured as life years gained. They found that patients receiving nimodipine had an average of 3.46 years of longer life expectancy compared with patients receiving placebo. The "cost-effectiveness" of one alternative option can be compared with that of the benchmark alternative by calculating the incremental cost-effectiveness ratio (ICER): [Cost $\left.\mathrm{Clternative}-\mathrm{Cost}_{\text {benchmark }}\right] /\left[\right.$ Outcome ${ }_{\text {alternative }}$ - Outcome benchmark ${ }^{22}$ In this study, the ICER was \$223 per life years gained; nimodipine increases patient life years at a low incremental cost..$^{20}$

As another example of CEA, Vuong and colleagues assessed the cost-effectiveness of 2 brain metastatic treatment modalities, namely resection versus stereotactic radiosurgery from the perspective of Germany's health system. ${ }^{31}$ For both treatment strategies, only costs of initial intervention and costs of retreatment (including repeat surgery, stereotactic radiosurgery, linear accelerator, or whole-brain radiotherapy) were calculated until death or 5.5 years of follow-up. The nonmonetary clinical outcome assessed was life year saved. In this study, use of stereotactic radiosurgery cost $€ 1683$ less than resection and increased the number of life years saved by 0.45 years. ${ }^{31}$ There are numerous examples of CEA in all neurosurgical subspecialties.

\section{Cost-Utility Analysis}

Cost-utility analysis follows the same framework as CEA. In fact, CUA is viewed by many economists as a subtype of CEA. The costs are expressed in monetary units. The outcomes are utility adjusted, where utility refers to a patient's preference for a particular health outcome. The outcome is expressed in quality-adjusted life years (QALYs), where the length of time spent in one health state is multiplied by the utility value of this health state (ranging from 0 [death] to 1 [perfect health]). Generic preference-based measurement systems such as the EuroQol 5 Dimension (EQ-5D) and the Short Form 6D (SF-6D) have facilitated the determination of the utility value. ${ }^{28}$ Cost-utility analysis presents cost per QALY gained by selecting one procedure or program over another. This type of economic evaluation is especially useful when mortality difference is not expected between interventions and/or quality of life is an important aspect of one treatment alternative. ${ }^{29}$ In comparison with CEA, CUA enables comparison of different interventions across numerous diseases, as a QALY has equal value irrespective of the nature of the alternative that delivers it. ${ }^{11}$ In North American studies, the cost/QALY threshold typically used to make resource allocation decisions varies from $\$ 50,000 /$ QALY to $\$ 100,000 /$ QALY. ${ }^{28}$ Figures of $£ 20,000$ to $£ 30,000$ per QALY are also used in the United Kingdom. ${ }^{6}$

Parker and colleagues questioned if minimally invasive versus open multilevel hemilaminectomy in patients with degenerative lumbar spinal stenosis was cost-effective. ${ }^{26}$ In this CUA, the authors described how they estimated the hospital costs, the physician costs, and indirect costs related to productivity losses. The authors used a

\section{TABLE 1: Summary of the 4 main complete economic evaluations}

\begin{tabular}{clll}
\hline $\begin{array}{c}\text { Type of Economic } \\
\text { Evaluation }\end{array}$ & \multicolumn{1}{c}{ Outcome Measure } & Cost Measure & \multicolumn{1}{c}{ Pearl } \\
\hline CMA & none (considered stable btwn alternatives) & monetary unit & need to be certain both alternatives provide the same outcome \\
CEA & any nonmonetary natural unit (disease specific) & monetary unit & inability to make interdisease comparison \\
CUA & utility score (nonmonetary) & monetary unit & capable to make interdisease comparison \\
CBA & monetary unit attributed to health effects & monetary unit & not frequently used in medicine \\
\hline
\end{tabular}


standard utility assessment, the EQ-5D, to estimate the QALYs before and at 2 years postprocedure. ${ }^{3,16}$ Although minimally invasive and open multilevel hemilaminectomy were associated with similar mean cost over 2 years (minimally invasive surgery: US\$23,109 vs open hemilaminectomy: US $\$ 25,420$ ), both treatment strategies provided equivalent improvement in QALYs (0.72) 2 years after surgery. ${ }^{26}$ Reporting findings of cost equivalence are of significance, as they may inform preconceived impressions of physicians.

As another example, Kumar and Rizvi evaluated the utility of spinal cord stimulation (SCS) and conventional medical management (CMM) compared with CMM alone for patients with multiple conditions including failed back surgery syndrome and complex regional pain syndrome. ${ }^{21}$ Outcome (QALY) was assessed at baseline and at 6 months using the EQ-5D. ${ }^{28}$ For each base case, the various categories of costs were tabulated for each treatment option (SCS+CMM vs CMM alone), including the cost of the implantable devices. In this study, the ICER for SCS+CMM was CAD\$9,293 (Canadian dollars) per QALY gained in failed back surgery syndrome and CAD $\$ 11,216$ per QALY gained in complex regional pain syndrome. ${ }^{21}$ Importantly, the ICER may vary between study groups, depending on the difference in QALYs between SCS+CMM versus CMM alone and the difference in cost between both treatment strategies.

Last, Ho and colleagues assessed the cost-effectiveness of decompressive craniectomy performed in patients with refractory intracranial hypertension after severe traumatic brain injury. ${ }^{13}$ The authors detailed which costs were tabulated and how QALY gained at the 18-month follow-up was calculated. This study showed that the average total hospital costs per QALY gained increased significantly when the predicted risk of a poor outcome was greater than $80 \%$ : US $\$ 682,000$ if predicted risk > $80 \%$ versus US $\$ 140,000$ if predicted risk $\leq 80 \%$, which is more than the usual accepted value of US $\$ 100,000$ deemed cost-effective. ${ }^{13}$ In trauma and oncology, the utility of a procedure can be difficult to rationalize solely on the information provided by economic evaluations (which integrate benefits and costs)., 914

\section{Cost-Benefit Analysis}

In CBA, the outcomes and the costs are expressed in monetary units. The human capital approach and the willingness to pay approach are 2 methods used to quantify in a monetary unit how much patients value improvement of their health (outcome). ${ }^{11}$ A person's willingness to pay may be influenced not only by personal preferences and values, but also by income and state of health. ${ }^{32}$ This type of economic evaluation is seldom used in the medical literature given the difficulty in placing a monetary value on various health states. ${ }^{29}$ To our knowledge, no study has reported a true CBA as described above regarding a neurosurgical treatment. Some papers include the key words "costs" and "benefit" in their titles but are in fact CEA.

True CBAs have been performed in the context of drug administration. Tang and colleagues conducted a cost-benefit analysis of administering ondansetron (Zofran) to prevent emesis in a high-risk patient population undergoing outpatient surgery. ${ }^{30}$ The overall weighted costs per patient for postoperative nausea and vomiting management were US\$21 $\pm \$ 7$. The authors used the "willingness to pay" method to attribute a monetary value of the benefits of preventing emesis. On average, patients were willing to pay US\$117 $\pm \$ 82$ to prevent postoperative nausea and vomiting if they underwent a similar surgery in the future. ${ }^{30}$ Dividing the assigned monetary value of "benefits" by the assigned cost results in the benefit-to-cost ratio, noted to be 5:1 in this study.

As a second example, Wasserfallen and colleagues assessed parents' willingness to pay for local anesthetics during blood sampling in children. ${ }^{32}$ The unit cost of the local anesthetic used in this study was $€ 5$.6. Among the various scenarios proposed, parents were willing to pay approximately $€ 4-€ 7$, testifying that the use of this drug has a high societal value..$^{32}$ This type of information, as noted by the authors, could be used to support billing of the drug to the parents through the health care facility or argue in favor of having the health care system absorb its cost as it delivers painless care.

\section{Existing Guidelines for Economic Evaluation Studies}

As neurosurgeons engage in the value paradigm, lead various economic evaluations, and share their findings with the scientific community, reviewing existing guidelines for economic submissions is of great value. Many societies and journals have described specific guidelines, most inspired by those put forth by Drummond and collaborators of the BMJ Economic Evaluation Working Party. ${ }^{8,17}$ They are useful for both authors and peer reviewers of economic submissions. ${ }^{8,17}$ A detailed review of the guidelines is beyond the scope of this overview. Table 2 summarizes key elements to be considered and reviewed when preparing either an economic evaluation or a cost analysis..$^{8,17,28}$

\section{Partial Economic Evaluation}

When outcomes are not considered in the analysis along with the cost data, the economic evaluation is qualified as partial. Studies that include a cost analysis report the cost incurred for either one specific part of the treatment (e.g., surgery-related costs), one phase of the episode of care (e.g., costs of inpatient care), or attempt to cost the entire episode of care.

The cost analysis is performed only for one procedure or program at one specific time. A partial economic evaluation in the form of cost analysis is the first step in conducting some form of complete economic evaluation.

Mendez and colleagues analyzed the costs of treatment of glioblastomas in Nova Scotia, Canada. ${ }^{25}$ This study was timely for 2 reasons: 1) Health care costs of illnesses with high mortality rates were, and remain, of interest in times of constrained health care resources. 2) At the time of publication, no information regarding cost of care of these patients existed in Canada. ${ }^{25}$ The authors provided a detailed description of their cost determination methodology and presented a breakdown of costs 
TABLE 2: Summary of key elements to review when preparing an economic evaluation or cost analysis

\begin{tabular}{|c|c|}
\hline \multicolumn{2}{|r|}{ Study Design } \\
\hline 1) study question & $\begin{array}{l}\text { State the economic importance. Address outcome and costs. Present the viewpoint (or perspective) of the eco- } \\
\text { nomic evaluation (i.e., patient, physician, institution, 3rd party payer, societal). }\end{array}$ \\
\hline 2) selection of alternative options & Describe the benchmark intervention \& the alternative intervention. \\
\hline 3) type of economic evaluation & State what type of economic evaluation is used \& justify its selection in relation to the study question. \\
\hline \multicolumn{2}{|r|}{ Data Collection } \\
\hline $\begin{array}{l}\text { 4) effectiveness data (clinical } \\
\text { outcome) }\end{array}$ & $\begin{array}{l}\text { If the effectiveness data are based on a single clinical trial, provide details of this study's design \& results. If the ef- } \\
\text { fectiveness data result from a summary of many studies, provide the method of data synthesis. If the effective- } \\
\text { ness data are insufficient, modeling can help supplement (e.g., decision-tree analysis, Markov model). }\end{array}$ \\
\hline $\begin{array}{l}\text { 5) "benefit" measurement \& } \\
\text { valuation }\end{array}$ & $\begin{array}{l}\text { State the primary outcome measure (see Table 1). Describe the method used for health benefits (if any value) } \\
\text { (e.g., trade off, standard gamble, contingent valuation). State if indirect benefits are measured \& describe their } \\
\text { relevance to the study question. }\end{array}$ \\
\hline 6) costing measurement & $\begin{array}{l}\text { Describe the provenance of cost data. Detail the methods of estimation of quantities of resources \& unit costs. } \\
\text { Provide details on adjustments for inflation, currency conversion, etc. }\end{array}$ \\
\hline 7) modeling & If modeling is used, justify its selection \& its parameters. \\
\hline \multicolumn{2}{|r|}{ Analysis \& Interpretation of Results } \\
\hline $\begin{array}{l}\text { 8) adjustments for timing of out- } \\
\text { comes \& costs }\end{array}$ & $\begin{array}{l}\text { Provide the time period over which outcomes \& costs are assessed. If discount rates are used when the study } \\
\text { period is }>1 \mathrm{yr} \text { ), provide the rates. If costs or outcomes are not discounted, provide justification. }\end{array}$ \\
\hline 9) allowance for uncertainty & $\begin{array}{l}\text { If any used, provide details on the statistical tests performed \& the confidence intervals around the main variables. } \\
\text { If a sensitivity analysis is performed, detail the approach used. }\end{array}$ \\
\hline 10) presentation of results & $\begin{array}{l}\text { Provide the answer to the study question. Provide the outcomes \& costs data separately before combining them in } \\
\text { an index or ratio (e.g., ICER). }\end{array}$ \\
\hline
\end{tabular}

incurred during the inpatient and outpatient care of glioblastoma patients. From the time of diagnosis to death, the mean cost of medical care was CAD $\$ 17,149$ per patient. The cost analysis enabled observation that highest costs were related to hospitalization with ward costs alone accounting for $48 \%$ of all costs. ${ }^{25}$

Once a potential area is identified for intervention, a CMA could be conducted between the benchmark approach and the proposed intervention assuming that the outcome is the same with both alternatives. Cost analysis can also be used to follow cost of treatment trends over time, especially when coupled with care redesign initiatives. $^{24}$

\section{Cost Determination for Economic Evaluations}

Cost information can be obtained from financial departments within health systems. Hospital-related costs and physician service costs may be managed by two distinct departments. Outside of a clinical trial, quantity of resources used and cost per unit of resource used should be based as much as possible on real patients included in the study. Physicians involved in health economics should build and nourish collaborations with their institution's finance department and learn to speak the same accounting language. They need to recognize the difference between costs and charges and understand the different subsidiary components of total costs.

The extent of cost determination is guided by the study question and whose perspective is being considered. Performing an economic evaluation from a patient's per- spective (narrowest) versus the society in general (broadest) will influence the extent of included costs. ${ }^{27}$ Although the societal perspective may be the gold standard, capturing the data on all costs incurred is nearly impossible. For this reason, and because provider cost data are more readily available, the provider's perspective is usually used.

\section{Hospital-Related Costs}

In the health care accounting setting, costs refer to the monetary value of resources used in performing an activity. Costs can be qualified as either direct versus indirect and variable versus fixed. Direct costs are defined as costs charged specifically to a revenue-producing cost center. Examples include radiology, laboratory, and pharmacy costs. These costs are most closely related to providing patient care and are most directly impacted by care redesign initiatives. Indirect costs are costs allocated to revenue-producing centers from nonrevenue departments, which support patient care. Examples include nutrition, administration, and patient escort. Of importance, the term "indirect cost" has a significance when used in an economic setting that is different from that in an accounting setting; it then includes changes in productivity resulting from morbidity (wait time, loss of earnings) or mortality. ${ }^{22}$ To prevent confusion, the term "productivity cost" is preferred.

Overall, there are 4 basic unit costs: variable direct, fixed direct, variable indirect, and fixed indirect. Variable costs refer to costs that change when volume changes versus fixed costs, which refer to costs that do not change when the volume changes. As an example, the cost of 
contrast is variable, as the more head CTs with contrast are done, the more the cost of this resource. However, the cost of the CT scanner is fixed, because the cost does not change when the number of head CT studies changes. In general, economists agree that cost analysis should preferentially use direct costs.

In the absence of cost data, various strategies have been developed to provide approximation of costs. The most frequent is to use interchangeably cost and charges. However, charges and costs are not equivalent. Charges refer to the expenses posted to a patient account for hospital services. Another common approximation for cost is the use of cost-to-charge ratios, which modifies the charges using a ratio reflecting historical proportional differences. Its interpretation is challenging: a low costto-charge ratio can represent either lower costs or higher charges. In addition, the cost-to-charge ratio can vary tremendously within the same institutions for different services. There is no substitute for cost data for economic evaluations.

\section{Physician Reimbursement (Not Cost)}

The physician reimbursement from Center for Medicare and Medicaid Services relies on appropriate coding for the diagnosis and the service provided. The reimbursement fee is based on the resource-based relative value scale. ${ }^{15}$ The relative value units are multiplied by a geographic practice cost index and are then converted to dollars by a conversion factor that changes annually. Similarly, private insurers have established various methodologies to calculate their own reimbursement fee schedule, with multiple influencing factors such as geographic location and regional demand for subspecialty.

Importantly, monetary values obtained for physician services are "reimbursement fees." This is different from the charges and also from the costs. As a general rule, the charges are the most expensive, the costs are the least expensive, and the reimbursement is somewhat in between. Adding hospital cost (or charges as a suboptimal surrogate for cost) and physician reimbursement, although not optimal, might be the closest to assess total cost of care delivery using patient-specific data.

\section{Costs From National Databases}

Depending on the study question and on access to cost data within the institution, the Nationwide Inpatient Sample (NIS) may be a resource for economic data. The NIS is one of the databases developed for the Healthcare Cost and Utilization Project (HCUP), sponsored by the Agency for Healthcare Research and Quality. The NIS approximates a $20 \%$ sample of discharges from HCUP-participating community hospitals within the US. The NIS contains clinical information such as age, sex, primary diagnosis (ICD-9 diagnosis), comorbidity measures, and length of stay. It also holds nonclinical information such as hospital characteristics (location, teaching/nonteaching, rural/community, and low/high volume) and expected payment source. The NIS provides total charges (not costs) for inpatient care only; it does not include charges (or costs) for physician services. In addition, costs related to outpatient clinic, rehabilitation, or long-term acute care hospitals are not included. Longitudinal studies are possible using the NIS database as it holds more than 20 years of data. However, this may be biased as only a subset of hospitals have been NIS members for multiple continuous years. Careful selection of data elements and preanalysis with descriptive statistics are essential to highlight missing data and hospital-specific data anomalies, as these can compromise the quality of the analysis. The University Health Consortium offers access to multicenter economic data to its members, using cost-to-charge ratios to generate their cost data. Crimson Continuum of Care, a business analytical tool, can also be used to analyze economic data. Each participating institution determines what subsidiary of cost is fed into the database (i.e., total cost, direct cost, or direct variable cost). Since different institutions may feed different types of cost, no comparison is feasible between participating institutions. Physicians using any type of database should understand well how it is built and how to extract meaningful and sound data.

\section{Costs for a Phase of Care Versus the Entire Episode of Care}

In studies reporting on complete economic evaluations or cost analysis, the cost determination is most often captured for a specific point of care or phase of care (e.g., preoperative outpatient, inpatient hospitalization, postoperative outpatient, and readmissions). However, there is a movement toward assessment of cost incurred to deliver care throughout the entire episode of care. It would be challenging to calculate the cost of the entire episode of care using current cost determination methods. The development of accountable care organizations will help facilitate this task by consolidating the care delivered to a patient population within one health system, using one constant costing method.

A promising accounting method called the time-driven activity-based costing method, developed by Kaplan et al., is being piloted in various specialties and institutions throughout the nation. ${ }^{18,19}$ It integrates the cost of each resource used in the process and the quantity of time the patient spends with each resource. ${ }^{18,19}$ This model has the potential to capture all costs (hospital- and physician-related costs) incurred throughout the episode of care using one consistent methodology. It is being piloted by some specialties to prepare bundle pricing estimates.

\section{Impact of Accessible Clinical Data on Economic Evaluations}

Health information technology has significantly spread over the last 2 decades; $59 \%$ of US hospitals now have at least a basic electronic health record..$^{2,5}$ In addition to not yet being widely adopted, modern health information technology systems are most often not used to their maximal capacity regarding communication, coordination, measurement, clinical decision support, and hardwiring of care pathway redesigns. Furthermore, current health care payment models, such as the fee-for-service model, might be counterproductive to health information technology's potential favorable economic impact, resulting in increased hospital charges. ${ }^{1,12}$ The same trap 
awaits all institutions as they transition from ICD-9 code sets to ICD-10 code sets, which requires more detailed documentation. From a national health care budget perspective, it will be key to mitigate the risk of increased charges due to changes in the documentation process (but not representing different care delivery). ${ }^{23}$ In the future, it may be pertinent to report in health economic analysis various payment models, electronic health record status, and coding system used, especially if 2 groups are compared. Overall, access to data, clinical or financial, is the first step. Physicians will need to have a comprehensive understanding of the data they are using to guide care redesign and obtain the greatest health benefits and cost savings. ${ }^{12,33}$

\section{Future Perspectives}

Economic evaluations and cost analysis are essential for the future of health care delivery. High-quality and methodologically sound economic evaluations are needed to confidently steer decision-making processes and care redesign initiatives toward optimal value of care. A multidisciplinary team should be coordinated, including economists, accountants, finance administrators, business professors, performance excellence specialists, and care providers. Although this alignment has been lacking in many health care institutions, those leading the path on health economics have understood the priceless value of collaborative teamwork performed by this new multidisciplinary team. Just like basic science developed a bench to bedside approach, health economics needs to develop a calculator to bedside approach, seeking to translate economic evaluation findings into the care delivery setting.

\section{Disclosure}

The authors report no conflict of interest concerning the materials or methods used in this study or the findings specified in this paper.

Author contributions to the study and manuscript preparation include the following. Conception and design: McLaughlin, Martin. Acquisition of data: McLaughlin. Analysis and interpretation of data: all authors. Drafting the article: McLaughlin. Critically revising the article: all authors. Reviewed submitted version of manuscript: McLaughlin, Ong, Tabbush, Martin. Approved the final version of the manuscript on behalf of all authors: McLaughlin. Study supervision: McLaughlin.

\section{References}

1. Abelson R, Creswell J, Palmer G: Medicare bills rise as records turn electronic. September 21, 2012. New York Times. (http:// www.nytimes.com/2012/09/22/business/medicare-billingrises-at-hospitals-with-electronic-records.html?smid=plshare) [Accessed September 14, 2014]

2. Adler-Milstein J, DesRoches CM, Furukawa MF, Worzala C, Charles D, Kralovec P, et al: More than half of US hospitals have at least a basic EHR, but stage 2 criteria remain challenging for most. Health Aff (Millwood) 33:1664-1671, 2014

3. Badia X, Diaz-Prieto A, Gorriz MT, Herdman M, Torrado H, Farrero E, et al: Using the EuroQol-5D to measure changes in quality of life 12 months after discharge from an intensive care unit. Intensive Care Med 27:1901-1907, 2001

4. Briggs AH, O'Brien BJ: The death of cost-minimization analysis? Health Econ 10:179-184, 2001
5. DesRoches CM, Charles D, Furukawa MF, Joshi MS, Kralovec P, Mostashari F, et al: Adoption of electronic health records grows rapidly, but fewer than half of US hospitals had at least a basic system in 2012. Health Aff (Millwood) 32: 1478-1485, 2013

6. Devlin N, Parkin D: Does NICE have a cost-effectiveness threshold and what other factors influence its decisions? A binary choice analysis. Health Econ 13:437-452, 2004

7. Douglas HR, Normand C: Economic evaluation: what does a nurse manager need to know? J Nurs Manag 13:419-427, 2005

8. Drummond MF, Jefferson TO: Guidelines for authors and peer reviewers of economic submissions to the BMJ. BMJ 313:275-283, 1996

9. Garau M, Shah KK, Mason AR, Wang Q, Towse A, Drummond MF: Using QALYs in cancer: a review of the methodological limitations. Pharmacoeconomics 29:673-685, 2011

10. Heaney DC, Shorvon SD, Sander JW: An economic appraisal of carbamazepine, lamotrigine, phenytoin and valproate as initial treatment in adults with newly diagnosed epilepsy. Epilepsia 39 (Suppl 3):S19-S25, 1998

11. Higgins AM, Harris AH: Health economic methods: costminimization, cost-effectiveness, cost-utility, and cost-benefit evaluations. Crit Care Clin 28:11-24, v, 2012

12. Hillestad R, Bigelow J, Bower A, Girosi F, Meili R, Scoville R, et al: Can electronic medical record systems transform health care? Potential health benefits, savings, and costs. Health Aff (Millwood) 24:1103-1117, 2005

13. Ho KM, Honeybul S, Lind CR, Gillett GR, Litton E: Costeffectiveness of decompressive craniectomy as a lifesaving rescue procedure for patients with severe traumatic brain injury. J Trauma 71:1637-1644, 2011

14. Honeybul S, Gillett GR, Ho KM, Lind CR: Neurotrauma and the rule of rescue. J Med Ethics 37:707-710, 2011

15. Hsiao WC, Couch NP, Causino N, Becker ER, Ketcham TR, Verrilli DK: Resource-based relative values for invasive procedures performed by eight surgical specialties. JAMA 260: 2418-2424, 1988

16. Jansson KA, Németh G, Granath F, Jönsson B, Blomqvist P: Health-related quality of life (EQ-5D) before and one year after surgery for lumbar spinal stenosis. J Bone Joint Surg Br 91:210-216, 2009

17. Jefferson T, Smith R, Yee Y, Drummond M, Pratt M, Gale $\mathrm{R}$ : Evaluating the BMJ guidelines for economic submissions: prospective audit of economic submissions to BMJ and The Lancet. JAMA 280:275-277, 1998

18. Kaplan RS: Improving value with TDABC. Healthe Financ Manage 68:76-83, 2014

19. Kaplan RS, Anderson SR: Time-driven activity-based costing. Harv Bus Rev 82:131-138, 150, 2004

20. Karinen P, Koivukangas P, Ohinmaa A, Koivukangas J, Ohman J: Cost-effectiveness analysis of nimodipine treatment after aneurysmal subarachnoid hemorrhage and surgery. Neurosurgery 45:780-785, 1999

21. Kumar K, Rizvi S: Cost-effectiveness of spinal cord stimulation therapy in management of chronic pain. Pain Med 14: 1631-1649, 2013

22. Kuper H, Jofre-Bonet M, Gilbert C: Economic evaluation for ophthalmologists. Ophthalmic Epidemiol 13:393-401, 2006

23. Levy B: ICD-10: 5 steps to a comprehensive financial impact analysis. Healthc Financ Manage 67:122-124, 126, 2013

24. McLaughlin N, Upadhyaya P, Buxey F, Martin NA: Valuebased neurosurgery: measuring and reducing the cost of microvascular decompression surgery. Clinical article. J Neurosurg 121:700-708, 2014

25. Mendez I, Jacobs P, MacDougall A, Schultz M: Treatment costs for glioblastoma multiforme in Nova Scotia. Can J Neurol Sci 28:61-65, 2001

26. Parker SL, Adogwa O, Davis BJ, Fulchiero E, Aaronson O, 


\section{Health care economics overview}

Cheng J, et al: Cost-utility analysis of minimally invasive versus open multilevel hemilaminectomy for lumbar stenosis. J Spinal Disord Tech 26:42-47, 2013

27. Robinson R: Economic evaluation and health care. What does it mean? BMJ 307:670-673, 1993

28. Rudmik L, Drummond M: Health economic evaluation: important principles and methodology. Laryngoscope 123:13411347,2013

29. Saint S, Chenoweth C, Fendrick AM: The role of economic evaluation in infection control. Am J Infect Control 29:338344,2001

30. Tang J, Wang B, White PF, Watcha MF, Qi J, Wender RH: The effect of timing of ondansetron administration on its efficacy, cost-effectiveness, and cost-benefit as a prophylactic antiemetic in the ambulatory setting. Anesth Analg 86:274-282, 1998

31. Vuong DA, Rades D, van Eck AT, Horstmann GA, Busse R: Comparing the cost-effectiveness of two brain metastasis treatment modalities from a payer's perspective: stereotactic radiosurgery versus surgical resection. Clin Neurol Neurosurg 115:276-284, 2013
32. Wasserfallen JB, Currat-Zweifel C, Cheseaux JJ, Hofer M, Fanconi S: Parents' willingness to pay for diminishing children's pain during blood sampling. Paediatr Anaesth 16:1118,2006

33. Zlabek JA, Wickus JW, Mathiason MA: Early cost and safety benefits of an inpatient electronic health record. J Am Med Inform Assoc 18:169-172, 2011

Manuscript submitted July 16, 2014.

Accepted August 19, 2014.

Please include this information when citing this paper: DOI: 10.3171/2014.8.FOCUS14455

Address correspondence to: Nancy McLaughlin, M.D., Ph.D., F.R.C.S.C., Department of Neurosurgery, David Geffen School of Medicine, 757 Westwood Plaza, Ste. 6236, Los Angeles, CA 900957436. email: nmclaughlin@mednet.ucla.edu. 